\title{
Management of Smoke Inhalational Laryngotracheal Injury: A Modest Experience
}

\author{
${ }^{1}$ Eswaran Venkata Raman, ${ }^{2}$ Vellore Thiruvengadam Anand, ${ }^{3}$ Ainippully Mohanan Anitha Kumari \\ ${ }^{4}$ Dhana Devi Thaoroijam, ${ }^{5}$ Chacko Jose
}

\begin{abstract}
Smoke inhalation injury (SII) to the airway is frequently seen in victims of accidental or contrived fires. However, with limited publications our knowledge and understanding about SII; and its management is just evolving over the last 50 years. Here, we discuss our experience with the challenges, we apprehended in managing survivors of SII with the objective of contributing to the existing guidelines of management.
\end{abstract}

Keywords: Inhalational injury, Endotracheal intubation, Stridor, Laryngotracheal stenosis.

How to cite this article: Raman EV, Anand VT, Kumari AMA, Thaoroijam DD, Jose C. Management of Smoke Inhalational Laryngotracheal Injury: A Modest Experience. Int J Otorhinolaryngol Clin 2015;7(2):60-63.

Source of support: Nil

Conflict of interest: None

\section{INTRODUCTION}

With advances in critical and emergency medicine, the survivors of burns and smoke inhalation injury (SII) have increased. Hence, we are now challenged with the delayed airway problems in the survivors. Smoke inhalation injury in burn patient independently increase mortality by $20 \%{ }^{1}$ Here, we share our experience with managing victims of SII after a fire accident.

Thirty-six victims arrived in our casualty after a fire broke out in an eight storied commercial building (none had external burns). Though the fire was promptly put out, early fumes ascended via ducts to the upper stories affecting several people trapped in higher levels. Out of 13 patients (Table 1) requiring ventilatory support four developed delayed airway complications.

\section{CASE REPORTS}

\section{Case I}

A 24 years old required ventilatory support for 4 days. The airway on discharge was edematous and congested

\footnotetext{
$1,2,5$ Senior Consultant, ${ }^{3}$ Consultant, ${ }^{4}$ Registrar

${ }^{1-4}$ Department of ENT, Manipal Hospital, Bengaluru, Karnataka, India ${ }^{5}$ Department of Emergency Medicine and Critical Care, Manipal Hospital, Bengaluru, Karnataka, India

Corresponding Author: Dhana Devi Thaoroijam, Registrar Department of ENT, Manipal Hospital, Bengaluru, Karnataka India, Phone: 9538365313, e-mail: dhana.th@gmail.com
}

with mobile but bulky cords. Two and half months later, he presented with stridor. Airway evaluation showed similar inflammatory status but cords fixed in paramedian position. Conservative management failed to relieve him, and hence a tracheotomy was done. Fiberoptic bronchoscopic (FOB) revealed posterior glottic stenosis with dense scar tissue fixing the vocal cords in adducted position. Stenotic segment was released followed by application of mitomycin C. Dilatation was repeated after 2 weeks.

Evaluation 6 months after the initial assault showed adequate airway and mobile vocal cords with marked reduction of the edema. He was successfully decannulated. He progressed well and last FOB showed mobile cords with $70 \%$ abduction.

\section{Case II}

A 31 years old required ventilatory support for 5 days. Fiberoptic bronchoscopic 4 months later revealed congested, bulky cords with restricted mobility and a $3 \mathrm{~cm}$ spiraling stenotic tracheal segment starting $2.5 \mathrm{~cm}$ below the vocal cords for which he underwent dilatation (Fig. 1).

A month later, he was tracheostomized for increasing stridor. Laryngotracheoplasty with anterior midline split of thyroid (below vocal cords), cricoid and cervical trachea up to stoma was performed and stented with montgomery T-tube. Six weeks later, T-tube was removed

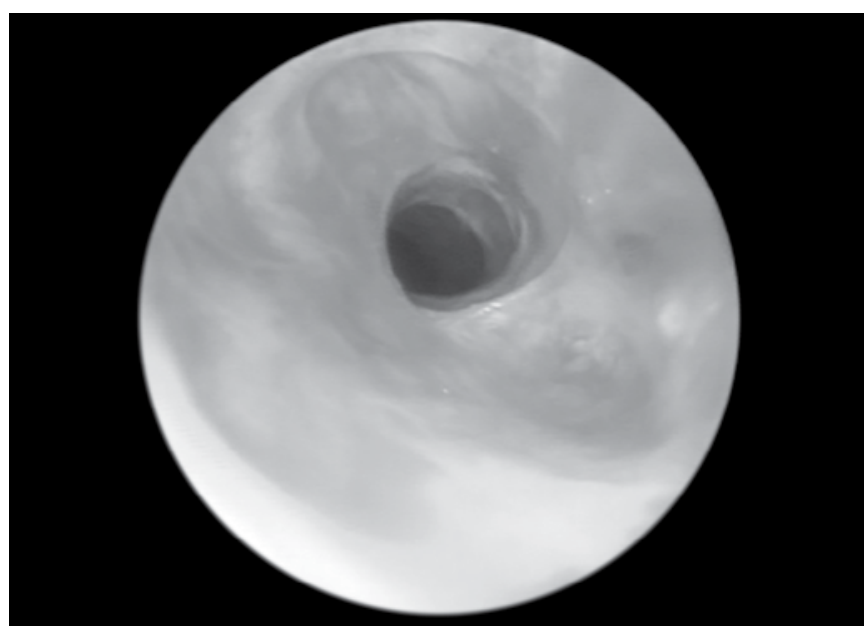

Fig. 1: Three centimeters spiralling stenosis extending from $2.5 \mathrm{~cm}$ below cords 
Table 1: Details of patients requiring airway support following inhalational trauma

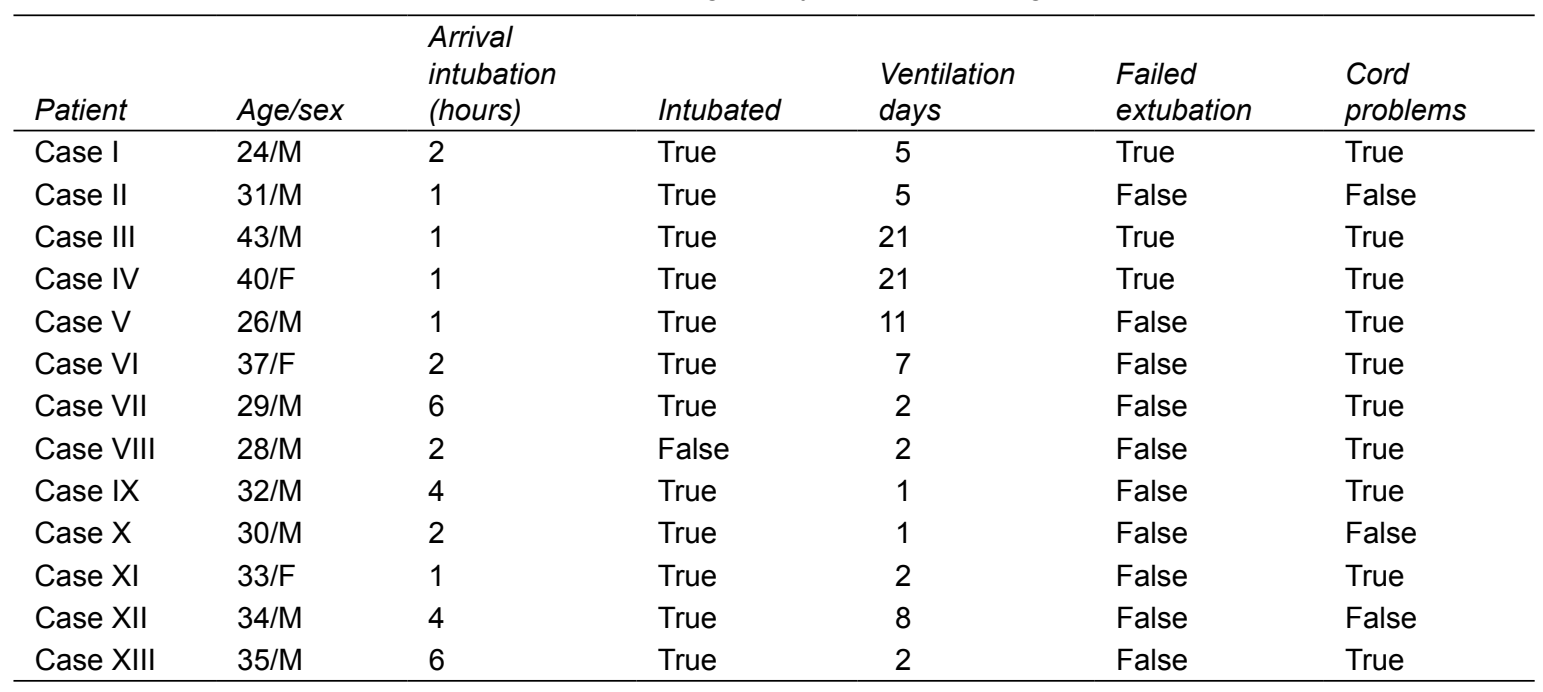

and airway reassessment showed stenosis at the level of glottis and subglottis. Tracheal lumen seemed adequate.

Laryngofissure with excision of the scar tissue with placement of Hood's laryngeal stent was done. Following removal of the laryngeal stent, it was observed that he had developed posterior glottic stenosis with cords fixed in midline. Subsequently, he underwent laryngotracheoplasty with posterior cricoid split, release of ankylosis of the cricoarytenoid joints and augmentation with cartilage graft and stenting with laryngotracheal mould. He is awaiting removal of the mould.

\section{Case III}

A 42 years old failed extubation on day 1 due to severe bronchorrhoea, requiring reintubation and ventilation for 3 weeks. He was tracheostomized in view of prolonged intubation and decannulated uneventfully prior to discharge. Fiberoptic bronchoscopic repeated after 6 months for stridor showed edematous larynx and a

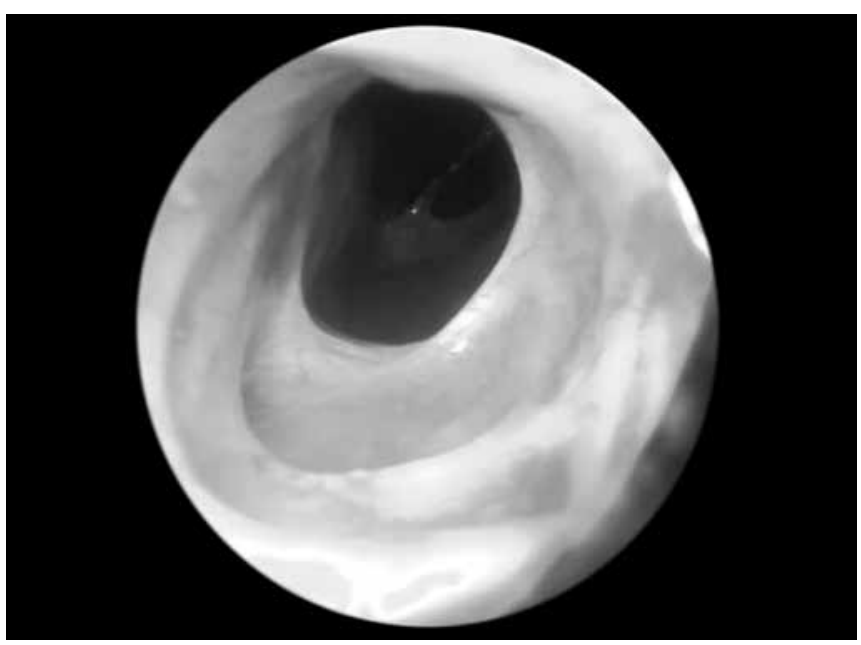

Fig. 2: Stenotic segment $5 \mathrm{~mm}$ thick, $2.5 \mathrm{~cm}$ from the vocal cords
$5 \mathrm{~mm}$ thick stenotic segment $2.5 \mathrm{~cm}$ from the vocal cords (Fig. 2). Three repeated dilatations were done in a span of 6 weeks. Each check FOB disclosed adamant restenosis. He underwent tracheal resection and reanastamosis at a different center. He has progressed well.

\section{Case IV}

This lady was tracheostomized after two failed attempts of extubation. Fiberoptic bronchoscopic showed severe edema and hyperemia with sloughing of the airway. Vocals cords were edematous with restricted mobility.

She continues to be tracheostomy dependent and symptomatic with exacerbation of breathlessness and hoarseness. Serial evaluation showed multiple level stenoses like case II. She has undergone several surgical procedures like T-tube stenting and laryngeal mould placement; modified Kashima and vocal cord lateralization in various institutes.

\section{DISCUSSION}

Otolaryngologists are involved in care of SII patients at various stages. Therefore, it is constructive to be appraised of the pathophysiology and principles of management of the condition.

In SII, the assault can be chemical or thermal. Steam is the carrier of heat, and hence cause for thermal injury. The airway is a good heat exchanger, hence thermal injury is limited to the upper airway involving the glottis. Subglottis, trachea and lung parenchyma are subject to chemical injury. The pathophysiology of SII is complex. It can be classified into three types based on the anatomic location. ${ }^{2}$ The first includes upper airway injuries caused mainly by thermal injury to mouth, oropharynx and larynx. The second type includes lower airway and parenchymal injuries (tracheal, bronchial and alveolar 
injuries) caused by chemical and particulate constituents of smoke. The third type includes metabolic asphyxiant constituents of smoke like carbon monoxide or hydrogen cyanide (CO and HCN). All three types of SII can occur individually or may co-exist making management more difficult and challenging.

Chemical injury is caused by products of incomplete combustion, like aldehydes and oxides of sulphur and nitrogen. Products of combustion of polyvinyl chlorides yield at least 75 toxic compounds including $\mathrm{CO}$ and $\mathrm{HCN}$. They impair oxygen delivery to, or use by, the tissues.

Fiberoptic bronchoscopic is imperative for diagnosis. However, it is not mandatory to make a definitive diagnosis of SII prior to securing the airway. Fiberoptic bronchoscopic in immediate 4 hours shows evolution of edema in tracheobronchial tree due to neutrophilic infiltration. Then, there is necrosis and sloughing of airway epithelium leading to full thickness ulceration of epithelial surface. Large portions of epithelial lining are shed and a pseudomembrane casts are formed that obstruct the airway. The lung parenchyma shows marked decrease in compliance, inactivation of surfactant leading to micro abscesses. In severe ARDS physiological shunting can occur. There is an exaggerated response seen immediately below the glottis due to eddies on the subglottic airflow with increase exposure to toxins making the respiratory epithelium below the vocal cords more susceptible than squamous epithelium above. ${ }^{3}$ It is ideal to repeat the examination after 24 to 48 hours. ${ }^{4}$ Biopsy has been advised when the diagnosis is uncertain. ${ }^{5}$

Orotracheal intubation is indicated for patients with poor mental status, airway obstruction and pulmonary failure. ${ }^{2}$ High peak inspiratory pressure and high cuff pressures are avoided thus, preventing ventilator induced lung injury. Early tracheostomy is done when prolonged ventilator support is anticipated. This prevents posterior glottic and subglottic stenosis which complicates and prolongs the management. It also facilitates pulmonary toileting.

The role of orotracheal intubation in SII are additive to the inhalational trauma. Stenosis was noticed in two studies after intubation. ${ }^{6,7}$ The pressure from the endotracheal tube and cuff independently expedite the sloughing and necrosis of the airway mucosa. Perichondritis and ankylosis of the cricoarytenoid joint complicate the situation. However, the fact that the stenosis and hypertrophic cicatrization occurring away from the intubation site explains that endotracheal tube and inhalational trauma potentiate their individual effects. The key difference of airway stenosis due to SII from stenosis due to other reasons is the intramural involvement in the inflammatory process. The capacity for hypertrophic scar proliferation is retained for extended period. The ongoing inflammatory process also affects vocal cord mobility mainly due to involvement of the cricoarytenoid joint.

The goals of management of delayed airway stenosis are to restore patency, to preserve satisfactory voice and to rid patient of the tracheostomy tube. To achieve these patients and the doctor must be prepared for an extensive period of treatment. The location of stenosis and the extent and degree of inflammation are important.

- If subglottis is normal, symptomatic trachea stenosis can be dilated serially. If this fails, T-tube insertion/ resection reanastamosis can be done after inflammation subsides.

- If the subglottis is involved a T-tube can be used with the upper limb passing just above the cords ${ }^{7}$ or in some cases grafting and stenting of subglottis is done. $^{8}$

- Stenosis of lower trachea and bronchi requires repeated dilation and are extremely difficult to treat. ${ }^{8}$ The optimal time for resection remains undetermined but delayed repair leads to fewer recurrences. ${ }^{9}$

- Management at the level of the glottis involves release of the glottic web and mobilization of the cricoarytenoid joint.

The successful outcome of treatment does not result in a normal airway. The quality of voice is diminished. And there are frequent episodes of respiratory infections. Patients are on long-term follow-up with FOB and PFT.

\section{CONCLUSION}

The extent and duration of the inflammatory response of the airway to inhalational injury is unpredictable. In case of inhalational airway injury, the norm is conservative management with minimal surgical intervention when buying time for inflammation to subside. Since studies clearly show the additive effect of trauma by orotracheal intubation to SII, we suggest early tracheostomy for those in whom assisted ventilation longer than 2 to 3 days is anticipated. This prevents posterior glottis and subglottic stenosis which would make tackling airway stenosis simpler. Survivors are counseled on prolonged duration of management and the final outcome of treatment in order to avoid patient dissatisfaction in the long run.

It is imperative that a well organized, protocol driven approach be brought into effective action so that right decisions are made at all stages of management starting from triage at the emergency room. This reduces the morbidity and mortality. The delayed complications would need customized management depending on the progress which can be assessed by regular follow-up and resorting to minimal surgical intervention when required. 


\section{REFERENCES}

1. Cancio LC, Pruitt BA Jr. Inhalation Injury. In: Tsokos GC, Atkins JL. Combat Medicine: Basic and Clinical Research in Military, Trauma and Emergency Medicine. Edition 1, US: Humana Press Inc; 2010.

2. Cancio LC. Airway management and smoke inhalation injury in burn patient. Clin Plastic Surg 2009;36(4):555-567.

3. Mallory TB, Brickley WJ. Pathology: with special reference to the pulmunory lesions. Ann Surg 1943;117(6):865-884.

4. Hunt JL, Agee RN, Pruitt BA Jr. Fiber optic bronchoscopy in acute inhalation injury. J Trauma 1975;15(8):641-649.

5. Masanes MJ, Legendre C, Lioret N, et al. Fiberoptic bronchoscopy for the early diagnosis of subglottal inhalation injury: comparative value in the assessment of prognosis. J Trauma 1994;36(1):59-67.

6. Adams C, Moison T, Chandrashekar AJ, Warpeha R. Endobronchial polyposis secondary to thermal inhalational injury. Chest 1979;75(5):643-645.

7. Whited RE. A prospective study of laryngotracheal sequelae in long-term intubation. Laryngoscope 1984;94(3):367-377.

8. Gaissert HA, Lofgren RH, Grillo HC. Upper airway compromise after inhalationa injury: complex structures of the larynx and trachea and their management. Ann Surg 1993 Nov;218(5):672-678.

9. Miller RP, Gray SD, Cotton RT, Myer CM. Airway reconstruction following laryngotracheal thermal trauma. Laryngoscope 1988;98(8 pt 1):826-829. 